Pacific Journal of Mathematics

QUASI-INJECTIVE MODULES AND STABLE TORSION 


\title{
QUASI-INJECTIVE MODULES AND STABLE TORSION CLASSES
}

\author{
E. P. ARMENDARIZ
}

In this note we examine the $\mathscr{T}$-torsion submodule of quasi-injective $R$-modules, $R$ a ring with unit, where $\mathscr{T}$ is a torsion class in the sense of $\mathrm{S}$. E. Dickson. We show that for a stable torsion class $\mathscr{T}$, the $\mathscr{T}$-torsion submodule of any quasi-injective module is a direct summand, while if $\mathscr{T}$ contains all Goldie-torsion modules, then every epimorphic image of a quasi-injective module has its $\mathscr{T}$-torsion submodule as a direct summand. In addition, we show that for a stable torsion class $\mathscr{T}$, all $\mathscr{T}$-torsion-free modules are injective if and only if $R=T(R) \oplus K$ (ring direct sum), with $K$ Artinian semisimple.

All $R$-modules will be unitary left $R$-modules. Originally our results were obtained for torsion classes closed under submodules. However, the referee has kindly pointed out how this assumption can be omitted throughout, supplying a proof in the case of Theorem 2.3. We take this opportunity to express our gratitude.

1. Following S. E. Dickson [2], a class $\mathscr{T}(\neq \varnothing)$ of $R$-modules is a torsion class if $\mathscr{T}$ is closed under factors, extensions, and arbitrary direct sums. The torsion class $\mathscr{T}$ is stable if $\mathscr{T}$ is closed under essential extensions. Every torsion class $\mathscr{T}$ determines in every $R$-module $A$ a unique maximal $\mathscr{T}$-submodule $T(A)$, the $\mathscr{T}$-torsion submodule of $A$, and $T(A / T(A))=0$, i.e., $A / T(A)$ is $\mathscr{T}$-torsion-free. The $R$-module $A$ splits if $T(A)$ is a direct summand of $A$. For further properties of torsion classes the reader is referred to [6].

The class $\mathscr{G}$ of Goldie-torsion modules is the smallest torsion class containing all factor modules $A / B$ where $B$ is essential in $A$, and their isomorphic copies. As shown in [1], $\mathscr{G}=\left\{A \mid Z_{2}(A)=A\right\}$ where $Z_{1}(A)=$ singular submodule of $A$ and $Z_{2}(A) / Z_{1}(A)=$ singular submodule of $A / Z_{1}(A)$ (see also [4]).

An $R$-module $A$ is quasi-injective provided every homomorphism from any submodule of $A$ into $A$ can be extended to an endomorphism of $A$. For any $R$-module $A, E(A)$ will denote the injective envelope of $A$.

2. The proof of the following is straightforward and so will be omitted.

Proposition 2.1. If the torsion class $\mathscr{T}$ is stable then every 
injective $R$-module splits. If $\mathscr{T}$ is closed under submodules, the converse holds and either condition is equivalent to $T(E(A))=E(T(A))$ for all $R$-modules $A$.

The next lemma can be found in [5, Proposition 2.3].

Lemma 2.2. If $A$ is a quasi-injective $R$-module and $E(A)=$ $M \oplus N$ then $A=(M \cap A) \oplus(N \cap A)$.

We now have

THEOREM 2.3. Let $\mathscr{T}$ be a stable torsion class. Then every quasi-injective $R$-module $A$ splits, $A=T(A) \oplus N$ where $N$ is quasiinjective and $\mathscr{T}$-torsion-free.

Proof. Choose a submodule $N$ of $A$ maximal with respect to $T(A) \cap N=0$. Then $E(A)=E(T(A)) \oplus E(N)$, hence by Lemma 2.2, $A=A \cap E(T(A)) \oplus A \cap E(N)$. Since $\mathscr{T}$ is stable $A \cap E(T(A))=T(A)$ and hence $A=T(A) \oplus N$ with $N=A \cap E(N)$ quasi-injective and $\mathscr{T}$ torsion-free.

Since the class $\mathscr{G}$ of Goldie-torsion modules is stable, it follows that $G(A)$ is a direct summand of $A$ whenever $A$ is quasi-injective; this was obtained by M. Harada in [5, Th. 1.7].

Let $\mathscr{T}$ be a torsion class; a submodule $B$ of an $R$-module $A$ is $\mathscr{T}$-closed if $T(A / B)=0$.

Lemma 2.4. Let $\mathscr{T}$ be a torsion class and let $B$ be a $\mathscr{T}$-closed submodule of the $R$-module $A$. If $M$ is any $R$-module and $f \in \operatorname{Hom}_{R}(M, A)$ then $N=f^{-1}(B)$ is $\mathscr{T}$-closed in $M$.

Proof. If $C / N$ is a $\mathscr{T}$-submodule of $M / N$ then $f(C) / B$ is a $\mathscr{T}$ submodule of $A / B$. Hence $f(C) \subseteq B$ since $B$ is $\mathscr{T}$-closed and so $N$ is $\mathscr{T}$-closed.

If $\mathscr{T}$ is a torsion class containing the class $\mathscr{G}$, then a $\mathscr{T}$-closed submodule $B$ of the $R$-module $A$ has no essential extension in $A$; hence if $A$ is quasi-injective then $B$ is a direct summand of $A$ by [3, Corollary 3 , p. 24]. Another way of showing this has been suggested by the referee: Choose $K$ maximal in $A$ with respect to $K \cap B=0$. Then $E(A)=E(K) \oplus E(B)$ so $A=(A \cap E(K)) \oplus(A \cap E(B))=K \oplus(A \cap E(B))$. Now $A / B \cong K \oplus(A \cap E(B)) / B$ and since $\mathscr{G} \cong \mathscr{T}, A \cap E(B)=B$.

ThEOREM 2.5. If $\mathscr{T}$ is a torsion class containing $\mathscr{G}$, and the $R$-module $A$ is an epimorphic image of a quasi-injective $R$-module then $A$ splits. 
Proof. Let $M$ be quasi-injective, $f: M \rightarrow A$ an epimorphism. Then $T(A)$ is a $\mathscr{T}$-closed submodule of $A$, hence by Lemma 2.4, $N=f^{-1}(T(A))$ is $\mathscr{T}$-closed in $M$. By the previous remark, $N$ is a direct summand of $M$, say $M=N \oplus P$. Then $f(P) \cap T(A)=0$ and so $A=T(A) \oplus f(P)$.

We note that the previous theorem is a generalization of $[7, T h$. 1.1] and the method employed is that of [8, Th. 2.10].

3. In [1, Th. 3.1] it was shown that a ring $R=G(R) \oplus K$ (ring direct sum), where $K$ is semisimple with minimum condition, if and only if all $\mathscr{C}$-torsion-free modules are injective. In this section we prove this result for any stable torsion class $\mathscr{T}$.

LEMMA 3.1. Let $R=S \oplus K$, where $S$ is semisimple with minimum condition. Then any $R$-module $A$ satisfying $K A=0$ is an injective R-module.

Proof. If $K A=0$ then $A$ is an injective $S$-module. Let $I$ be a left ideal of $R$; then $I=S_{1} \oplus K_{1}$ where $S_{1} \subseteq S, K_{1} \subseteq K$ are left ideals of $R$. Also $1=u+v, u \in S, v \in K$. If $f: I \rightarrow A$ is an $R$-homomorphism, then for any $b \in K_{1}, 0=v f(b)=f(b)$. There is an $S$-homomorphism $g: S \rightarrow A$ coinciding with $f$ on $I$, and this yields an $R$-homomorphism $g^{*}: R \rightarrow A$ coinciding with $f$ on $I$ if we define $g^{*}(s+k)=g^{*}(s)$.

THEOREM 3.2. Let $\mathscr{T}$ be a stable torsion class. Then all $\mathscr{T}-$ torsion-free $R$-modules are injective if and only if $R=T(R) \oplus K$, where $K$ is a semisimple ring with minimum condition.

Proof. Assume $A$ is injective whenever $T(A)=0$. Since the class $\mathscr{F}$ of $\mathscr{T}$-torsion-free modules is closed under submodules [2], every submodule of any $A \in \mathscr{F}$ is injective, hence is a direct summand, and so every $A \in \mathscr{F}$ is completely reducible. Let $M$ be any $R$-module and assume $T(M) \neq 0$. If $T(M)$ is essential in $M$ then $T(M)=M$, since $\mathscr{T}$ is stable. Otherwise select $B$ maximal relative to $B \cap T(M)=0$. Then $T(B)=0$ and so $B$ is injective. Thus $M=B \oplus U$. Now $M / U \cong B \in \mathscr{F}$ so that $T(M) \cong U$ by [2, Proposition 2.4]. The maximal property of $B$ ensures that $T(M)$ is essential in $U$ and so $U=$ $T(M)$. In particular $R=T(R) \oplus K$, where $K$ is a completely reducible $R$-module since $K \in \mathscr{F}$. The decomposition is two-sided since right multiplications are $R$-homomorphisms and both classes $\mathscr{T}$ and $\mathscr{F}$ are closed under factors.

Conversely, assume $R=T(R) \oplus K$, where $K$ is a semi-simple ring with minimum condition. We note that for any $R$-module $A$, if $T(A)=$ 0 then $T(R) A=0$. For if $A \in \mathscr{F}$ and $0 \neq a \in A$ then $T(R) \alpha$ is an epimorphic image of $T(R)$ and so $T(R) a \subseteq T(A)=0$. That every $\mathscr{T}$ torsion-free module is injective now follows from Lemma 3.1. 
We conclude with the following example. Let $R$ be the ring of lower triangular $2 \times 2$ matrices over a finite field and let $\mathscr{T}$ be the smallest torsion class containing all projective simple $R$-modules. Note that $\mathscr{T}$ contains nonzero $R$-modules since every simple in the socle of $R$ is in $\mathscr{T}$. Moreover $R$ is a hereditary Artinian ring with (rad $R)^{2}=0$ so by [9, Theorem $\mathrm{B}$ ] every nonprojective simple is injective. Since $R$ is not semisimple, it has nonzero $\mathscr{T}$-torsion-free $R$-modules. If $T(A)=0$ for an $R$-module $A \neq 0$ then socle $(A)$ contains no projective simples. Since $R$ is Noetherian and socle $(A)$ is essential in $A, A$ is injective. It is readily verified that socle $(R)=T(R)$. Thus the condition that $T$ be stable is needed in Theorem 3.2, even when $T$ is closed under submodules.

\section{REFERENCES}

1. J. S. Alin and S. E. Dickson Goldie's torsion theory and its derived functor, Pacific

J. Math. 24 (1968), 195-203.

2. S. E. Dickson, A torsion theory for Abelian categories, Trans. Amer. Math. Soc. 121 (1966), 223-235.

3. C. Faith, Lectures on injective modules and quotient rings, 49, Springer-Verlag, Berlin, 1967.

4. A. W. Goldie, Torsion free modules and rings, J. Algebra 1 (1964), 268-287.

5. M. Harada, Note on quasi-injective modules, Osaka J. Math. 2 (1965), 351-356.

6. J. P. Jans, Some aspects of torsion, Pacific J. Math. 15 (1965), 1249-1259.

7. E. Matlis, Divisible modules, Proc. Amer. Math. Soc. 11 (1960), 385-391.

8. F. L. Sandomierski, Semisimple maximal quotient rings, Trans. Amer. Math. Soc. 128 (1967), 112-120.

9. A. Zaks, Simple modules and hereditary rings, Pacific J. Math. 26 (1968), 627-630.

Received October 7, 1968.

UNiversity of TeXas

Austin, Texas 


\section{PACIFIC JOURNAL OF MATHEMATICS}

\section{EDITORS}

\author{
H. ROYDEN \\ Stanford University \\ Stanford, California \\ Richard Pierce \\ University of Washington \\ Seattle, Washington 98105
}

\author{
J. DugundJI \\ Department of Mathematics \\ University of Southern California \\ Los Angeles, California 90007 \\ BASIL GoRDON \\ University of California \\ Los Angeles, California 90024
}

\section{ASSOCIATE EDITORS}
E. F. BECKENBACH
B. H. Neumann
F. WOLF
K. YoSHIDA

\section{SUPPORTING INSTITUTIONS}

\author{
UNIVERSITY OF BRITISH COLUMBIA \\ CALIFORNIA INSTITUTE OF TECHNOLOGY \\ UNIVERSITY OF CALIFORNIA \\ MONTANA STATE UNIVERSITY \\ UNIVERSITY OF NEVADA \\ NEW MEXICO STATE UNIVERSITY \\ OREGON STATE UNIVERSITY \\ UNIVERSITY OF OREGON \\ OSAKA UNIVERSITY \\ UNIVERSITY OF SOUTHERN CALIFORNIA
}

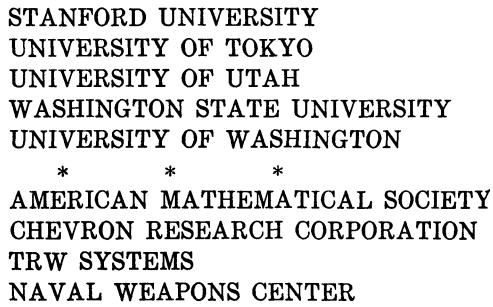

The Supporting Institutions listed above contribute to the cost of publication of this Journal, but they are not owners or publishers and have no responsibility for its content or policies.

Mathematical papers intended for publication in the Pacific Journal of Mathematics should be in typed form or offset-reproduced, double spaced with large margins. Underline Greek letters in red, German in green, and script in blue. The first paragraph or two must be capable of being used separately as a synopsis of the entire paper. It should not contain references to the bibliography. Manuscripts, in duplicate if possible, may be sent to any one of the four editors. Please classify according to the scheme of Math. Rev. 36, 1539-1546. All other communications to the editors should be addressed to the managing editor, Richard Arens, University of California, Los Angeles, California, 90024.

50 reprints are provided free for each article; additional copies may be obtained at cost in multiples of 50 .

The Pacific Journal of Mathematics is published monthly. Effective with Volume 16 the price per volume (3 numbers) is $\$ 8.00$; single issues, $\$ 3.00$. Special price for current issues to individual faculty members of supporting institutions and to individual members of the American Mathematical Society: $\$ 4.00$ per volume; single issues $\$ 1.50$. Back numbers are available.

Subscriptions, orders for back numbers, and changes of address should be sent to Pacific Journal of Mathematics, 103 Highland Boulevard, Berkeley, California, 94708.

PUBLISHED BY PACIFIC JOURNAL OF MATHEMATICS, A NON-PROFIT CORPORATION

Printed at Kokusai Bunken Insatsusha (International Academic Printing Co., Ltd.), 7-17, Fujimi 2-chome, Chiyoda-ku, Tokyo, Japan. 


\section{Pacific Journal of Mathematics}

\section{Vol. 31, No. $2 \quad$ December, 1969}

Efraim Pacillas Armendariz, Quasi-injective modules and stable torsion

classes..........................................

J. Adrian (John) Bondy, On Ulam's conjecture for separable graphs...

Vasily Cateforis and Francis Louis Sandomierski, On commutative rings over which the singular submodule is a direct summand for every module .....

Rafael Van Severen Chacon, Approximation of transformations with continuous

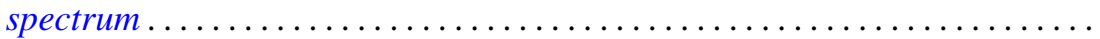

Raymond Frank Dickman and Alan Zame, Functionally compact spaces ...... 303

Ronald George Douglas and Walter Rudin, Approximation by inner

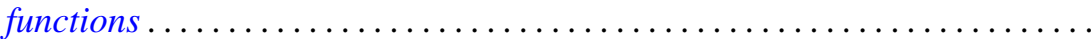

John Walter Duke, A note on the similarity of matrix and its conjugate

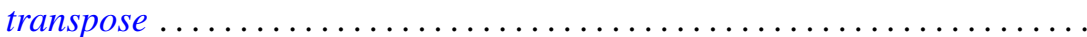

Micheal Neal Dyer and Allan John Sieradski, Coverings of mapping

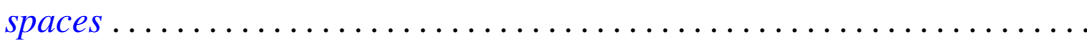

Donald Campbell Dykes, Weakly hypercentral subgroups of finite groups .....

Nancy Dykes, Mappings and realcompact spaces.....................

Edmund H. Feller and Richard Laham Gantos, Completely injective

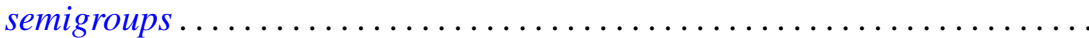

Irving Leonard Glicksberg, Semi-square-summable Fourier-Stieltjes

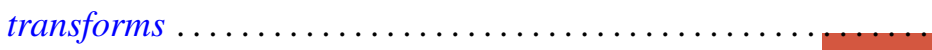

Samuel Irving Goldberg and Kentaro Yano, Integrability of almost cosymplectic structures...

Seymour Haber and Charles Freeman Osgood, On the sum $\sum\langle n \alpha\rangle^{-t}$ and numerical integration ..........................

Sav Roman Harasymiv, Dilations of rapidly decreasing functions ....

William Leonard Harkness and R. Shantaram, Convergence of a sequence of

transformations of distribution functions

Herbert Frederick Kreimer, Jr., A note on the outer Galois theory of rings ...

James Donald Kuelbs, Abstract Wiener spaces and applications to analysis. .

Roland Edwin Larson, Minimal $T_{0}$-spaces and minimal $T_{D}$-spaces...

A. Meir and Ambikeshwar Sharma, On Ilyeff's conjecture .

Isaac Namioka and Robert Ralph Phelps, Tensor products of compact convex sets....

James L. Rovnyak, On the theory of unbounded Toeplitz operators ....

Benjamin L. Schwartz, Infinite self-interchange graphs.......

George Szeto, On the Brauer splitting theorem...

Takayuki Tamura, Semigroups satisfying identity $x y=f(x$,

Kenneth Tolo, Factorizable semigroups .................. 